\title{
Superconductor consortium approaches test
}

\section{Washington}

NEXT month marks the second birthday of the Consortium for Superconducting Electronics, an ambitious attempt by four leading US research institutions to translate scientific prowess into commercial advantage. Two years ago, the move seemed to be just what the doctor ordered to help the United States compete with Japan in developing applications of high-temperature superconductors. Earlier in 1989, a committee to advise President George Bush on high-temperature superconductors - known as the 'wise men's committee' - had recommended the formation of just such consortia to combat a US tendency to focus on short-term goals and neglect research that is still far from commercialization. Hopes and expectations were high.

But after the initial fanfare accompanying the creation of the consortium, which includes IBM, AT\&T Bell Labs, the Massachusetts Institute of Technology (MIT) and MIT's Lincoln Laboratory, it virtually sank from sight. What has it been doing?

The two years have been a quiet success, says Richard Ralston of Lincoln Lab, the principal director of the consortium. "We have met all of our first-year [technical] goals and are on a strong track to meet all the second-year goals." The major frustration has been the lack of a small, aggressive firm in the consortium that would push hard for quick commercialization of products from the collaboration's research, but that is likely to be corrected within the month. Although it is still too early to say just how much the consortium will help US industry compete in the coming superconductor market, its participants say this economic experiment has already offered valuable insight into the right way and the wrong way to run a consortium, and its success or failure is likely to influence greatly the formation of such collaborations in the future.

The consortium is founded on complete openness in research and the "full sharing of intellectual property", Ralston says. The charter of the consortium allows each member full rights to commercialize any products that arise from research done in the consortium; furthermore, members agree to give each other rights to research done before the consortium was formed, although the members can charge royalties for previous work.

"The beauty of this consortium is that we basically got upper management out of the way, and scientists could talk to each other," says Bob Dynes, who served as Bell Labs' director for the consortium

until he moved to the University of California, San Diego at the beginning of this year. "Scientists don't care about these barriers [erected by businesses to protect their own interests]."

Nonetheless, Ralston says, it was tricky at first to get researchers to accept the idea of sharing everything — especially researchers at IBM and Bell Labs, two traditional rivals with corporate cultures that emphasize the protection of property rights. But the consortium "broke the ice" by using MIT and Lincoln Lab as neutral ground and by choosing the initial projects carefully so that researchers saw the value of working together and trading on each of working together and trading on each

potential. But the two years of running the consortium have produced a wealth of experience that could prove valuable to others contemplating such a move.

The glue that holds the consortium together is the graduate students, Ralston says. University faculty and laboratory scientists visit back and forth, usually for a day at a time, but they generally have commitments that keep them close to home. The graduate students, on the other hand, will spend weeks or months away. One of Kastner's students at MIT, for example, goes to IBM to fabricate his devices and to AT\&T Bell Labs for measurements, "so he actually shuttles between the three labs," Kastner says. "It's good for the students - people at the labs give them a lot of attention."

It's also good for the consortium. It is often the students who figure out how to use the resources at the different locations, says William Gallagher, IBM's director for the consortium. The 'graduate student glue' is one major advantage over consortia that do not include universities.

One of the biggest advantages of having different institutions interacting freely on research is the coming together of diverse

other's strengths.

The result: "There have been a lot of important technological steps," says Marc Kastner, the MIT director for the consortium, although there has been "nothing really earth-shaking, which is why you haven't heard anything [in the press]." The success story that best illustrates the advantages of the consortium is the development of electronic devices made from a 'trilayer' comprising two layers of niobium, a low-temperature superconductor, sandwiched around a layer of aluminium oxide, an insulator. Lincoln Lab developed the technology for growing the trilayers on a small scale; AT\&T grew the trilayers on the large silicon wafers it uses for making electronic circuits; and IBM put the wafers on one of its processing lines, where it etched the trilayers into millions of electronic circuits.

Each of the institutions contributed something the others did not have: Lincoln Lab, a technique for growing the trilayers; AT\&T, a facility for working with materials on a large-scale; and IBM, state-of-the-art processing equipment for carving microscopic circuits. The result is that IBM can now turn out made-to-order superconducting circuits on an almostcommercial scale, allowing researchers in the consortium to develop and test new devices.

For now, however, the technological value of the consortium is still mostly research cultures, says Bertram Batlogg, who succeeded Dynes as head of Bell Labs's consortium work. The consortium has allowed the scientists to "learn different ways science and research is done", Batlogg says.

Perhaps the biggest lesson the participants have learned from this collaborative effort concerns something that was initially left out of the mix. With two major industrial labs, a top-notch research university and a government lab, it seemed as if the Consortium for Superconducting Electronics had everything it needed, but experience has proven otherwise.

"What's missing are small commercial companies," Dynes says. The consortium will not have finished its job until its collaborative research has been turned into commercial products, and small firms are the ones that historically have brought cutting-edge technology to the marketplace in the United States. IBM and AT\& T are big companies that will not devote a great deal of their attention to new, unproven technologies and that typically take several years to commercialize products, Dynes says, so the consortium really needs "companies whose lives depend on" turning research into results. "That really focuses their attention."

The consortium should fill in this gap in the next few weeks, Ralston says. It has been negotiating with a small superconductor company for about six months, and 
only a few small details remain to be settled.

Somewhat surprisingly, these negotiations have taken as long as the original talks between the four initial members of the consortium. "We've learned that it's easier for two large corporations to agree on something than for a large corporation and a small corporation," Dynes says. The problem, Ralston says, is that "patent rights are more important to small start-ups because they don't have a market yet rights are a large part of their portfolios. That has made for some rather extensive negotiations."

The lack of a small company has contributed to a second difficulty for the consortium. When it was formed, the consortium was counting on funding of between $\$ 4$ million and $\$ 6$ million from the Defense Advanced Research Projects Agency (DARPA). But DARPA's money has been coming much more slowly than expected, preventing Lincoln Lab from buying some of the equipment it needs, and the defence agency has so far committed itself to only two years of funding, with an option for a third, while the consortium is planned to last for at least a decade.

One of the things DARPA is waiting for, Ralston says, is to see if the consortium can accommodate small companies. "[DARPA] is not yet convinced the consortium has shown that technical progress can be translated to products, which is what DARPA wants," he says. One question that the agency would like answered is, "How open will the big guys be to letting a small company commercialize the technology?"

In many ways, the Consortium for Superconducting Electronics is likely to serve as a test case for the value of research consortia in general. Ralph Gomory, director of the Sloan Foundation and one of the principal authors of the 'wise men's report on superconductivity' when he was at IBM three years ago, argues that consortia are useful only in certain special cases, mostly in fields that are early in their development where relatively small amounts of money spent intelligently can make a big difference several years on. "High-temperature superconductors offer about as good a case for consortia as you're ever likely to see," he says.

If the Consortium for Superconducting Electronics, with its membership drawn from the cream of US research institutions, does not make a difference to US competitiveness in superconducting applications over the next decade, it will be hard to make the case for other consortia. If, on the other hand, this collaboration proves a success, it could well become a model for others. It is still too early to tell, but with the coming addition of a small company to the consortium, its ability to bring new products to the marketplace will soon be tested.

\section{Go-ahead for Centocor}

\section{Washington}

IN the highly publicized race to market in the United States the first monoclonal antibody for therapeutic use, Centocor surged ahead of rival biotechnology company Xoma last week, when an advisory committee to the Food and Drug Administration (FDA) recommended that Centocor's new drug for the treatment of Gram-negative bacterial infections and septic shock be approved for marketing in the United States. Xoma was dealt a severe blow when the committee announced that although it would hear Xoma's presentation, it would not make a recommendation at that time. News of the panel's decision sent Centocor's stock climbing and Xoma's plummeting and could give Centocor the edge in the marketplace.

Sepsis, the leading cause of morbidity and death in hospitalized patients, with an incidence of more than 400,000 cases each year in the United States, is most commonly caused by Gram-negative bacteria, such as Escherichia coli. Antibiotics, while able to destroy bacteria, have no effect on the endotoxins released into the bloodstream by such Gram-negative bacteria. These endotoxins can lead to septic shock, organ failure and death, often in a matter of hours. Centoxin, Centocor's human monoclonal antibody, binds to endotoxins released by Gram-negative bacteria, neutralizing their effects.

In a placebo-controlled clinical trial, Centocor enrolled 543 patients with sepsis and a suspected diagnosis of Gram-negative bacteraemia. Although Centoxin produced no significant benefit in the treatment group as a whole, use of the drug among a subset of patients whose blood contained Gram-negative bacteria reduced mortality by 39 per cent. The effect was even more pronounced in patients who were also in septic shock.

Although Centoxin is already approved in the Netherlands, Germany, Luxembourg, Denmark and the United Kingdom, several panel members were less than enthusiastic about the preclinical and clinical data presented by Centocor. The results are "suggestive but marginal", said Donald Summers, a panel member and professor and chairman in the department of cellular, viral and molecular biology at the University of Utah Medical Center.

One of the main criticisms levelled at Centocor was that only patients later confirmed as having Gram-negative bacteria in the bloodstream are likely to benefit from Centoxin. As this diagnosis can be made only after the event, physicians would need to prescribe Centoxin on the basis of a presumptive rather than a definitive diagnosis, which some panel members felt could lead to the treatment of many patients without the Gram-negative bacteria who would derive no benefit.

Unlike Centoxin, Xoma's anti-endotoxin monoclonal antibody, Xomen-E5, is a mouse-derived antibody. In two separate clinical trials, Xomen-E5 had a significant life-saving benefit in a slightly different subset of patients - that is, in those patients with confirmed Gram-negative infections and organ failure but who were not in shock. Xoma was not asked to conduct further trials, but FDA officials said they were unable to make a thorough review of the second study in time for last week's meeting.

\section{Nuclear cleanup costs rise}

\section{Washington}

THE US Department of Energy (DOE) will need about $\$ 12,000$ million more to clean up its nuclear weapons complex for the years 1993 to 1997 than it is likely to receive in funding, according to a DOE report issued last week.

In 1989, the department committed itself to a 30-year programme to clean up all active and inactive sites in the complex, and each year it offers an updated fiveyear plan. In its new plan, the DOE estimates that it will cost $\$ 40,700$ million from 1993 to 1997 to get rid of immediate hazards, to carry out cleanup agreements already made by the DOE and to ensure compliance with environmental regulations.

However, under last year's congressional budget agreement, funding for the DOE cleanup is scheduled to rise by only
10 per cent a year over that period, and in that case the department will receive only $\$ 28,700$ million from 1993 to 1997.

How the department will extricate itself from this bind is not clear. Under current budget constraints, Congress would have to pull the extra money from other programmes, and the rising cleanup costs are already putting a strain on DOE's other missions (see, for example, Nature 353, 6; 5 September 1991). Even if it were to receive the additional funds, the DOE has said it may not be able to spend them effectively. On the other hand, if DOE does not spend the money, it may find itself breaking the law with respect to environmental regulations or breaking agreements with the Environmental Protection Agency and various state governments. 